\title{
Biodegradation and biodetoxification of Fusarium mycotoxins by Sphaerodes mycoparasitica
}

Seon Hwa Kim and Vladimir Vujanovic*

\begin{abstract}
A fungus Sphaerodes mycoparasitica SMCD 2220-01 is a host specific mycoparasite against plant pathogenic Fusarium species. Fusarium spp. are producing a plethora of mycotoxins including zearalenone (ZEN), deoxynivalenol (DON) and its acetylated derivatives, 3-acetyl-deoxynivalenol (3-ADON) and 15-acetyl-deoxynivalenol (15-ADON). The SMCD 2220-01 strain substantially reduced DON, 3-ADON, 15-ADON, and ZEN production capacity in co-culture system. Degradation and detoxification of the pure mycotoxins were also achieved when exposed to SMCD 2220-01 in shake flasks. The thin layer chromatography (TLC) combined with high performance liquid chromatography-electrospray ionization-high resolution mass spectrometry (HPLC-ESI-HRMS) revealed that the amount of mycotoxins exposed to SMCD 2220-01 was considerably reduced compared to control. ZEN level was decreased by 97\%, while zearalenone sulfate $\left(\left[\mathrm{M}-\mathrm{H}+\mathrm{SO}_{3}\right]^{-}\right.$at $\left.\mathrm{m} / \mathrm{z} 397.1052 \mathrm{C}_{18} \mathrm{H}_{21} \mathrm{O}_{8} \mathrm{~S}_{1}\right)$ was detected as a metabolite of ZEN converted to less toxic molecule by the mycoparasite. Further, the mycoparasite appeared to degrade DON, 3-ADON, and 15-ADON by 89, 58, and $72 \%$, respectively. The deoxynivalenol sulfate $\left(\left[\mathrm{M}-\mathrm{COCH}_{3}+\mathrm{SO}_{3}-\mathrm{CH}_{2} \mathrm{O}\right]^{-}\right.$at $\left.\mathrm{m} / 2345.2300 \mathrm{C}_{14} \mathrm{H}_{17} \mathrm{O}_{8} \mathrm{~S}_{1}\right)$ was detected as a less toxic metabolic product of DON and 3-ADON. These findings report the SMCD 2220-01 effectiveness to lower mycotoxins-producing capacities of Fusarium, degrade pure mycotoxins and transform them to less toxic metabolites, opening new opportunities for research and innovation for detoxification of mycotoxins.
\end{abstract}

Keywords: Mycotoxins, Fusarium, Biocontrol, Biodegradation, Biotransformation, Biodetoxification

\section{Introduction}

Fusarium graminearum, Fusarium culmorum and Fusarium avenaceum are the causal agent of Fusarium head blight (FHB) of small grain cereals in fields worldwide (Kim and Vujanovic 2016). These Fusarium species can produce various secondary metabolites including toxins such as zearalenone (ZEN), deoxynivalenol (DON/ vomitoxin), 3-acetyl-deoxynivalenol (3-ADON), 15-acetyldeoxynivalenol (15-ADON) and aurofusarin (AUR). ZEN, a member of the resorcyclic acid lactone family, is a known hydrophobic mycotoxin produced by $F$ graminearum and F. culmorum (Caldwell et al. 1970; Katzenellenbogen et al. 1979). The toxicity of ZEN is mainly derived from its lactone ring and free C-4 hydroxyl group (El-Sharkawy

\footnotetext{
*Correspondence: vladimir.vujanovic@usask.ca Department of Food and Bioproduct Sciences, University of Saskatchewan, 51 Campus Drive, Saskatoon, SK S7N 5A8, Canada
}

and Abul-Hajj 1988a). ZEN contamination found in maize and wheat grains, food and feed, poses a threat to health of humans and animals due to its similar chemical structure to estrogen (Iqbal et al. 2014; Shier et al. 2001). DON, 3-ADON, and 15-ADON, belonging to Type B trichothecenes, in which the 12,13-epoxy ring is essential for their cytotoxicity (Zhou et al. 2008); it was revealed as inhibitor of eukaryotic protein synthesis as well as RNA and DNA synthesis (Ehrlich and Daigle 1987; Hussein and Brasel 2001; Middlebrook and Leatherman 1989). The toxicity of DON and its derivatives, 15-ADON and 3-ADON, were demonstrated by using the 5-bromo-2'-deoxyuridine incorporation assay assessing DNA-synthesis (Sundstøl Eriksen et al. 2004), in which there may be a link between degradation process and resistance to the mycotoxins.

Mycotoxin-degrading microbes have been isolated mainly from agriculture crops and plant production environments. Sphaerodes mycoparasitica SMCD 2220-01 is 
the fungus originally isolated from wheat and asparagus fields in association with $F$. graminearum, F. oxysporum and F. avenaceum (Vujanovic and Goh 2009). SMCD 2220-01 shown mycoparasitic lifestyle and is promising biological control agent against mycotoxin producing Fusarium pathogens (Vujanovic and Goh 2010; Kim and Vujanovic 2016). In addition to the biocontrol effect, SMCD 2220-01 was effective in reducing AUR mycotoxin production in red pigmented Fusaria by down-regulating AUR gene expression (Vujanovic and Goh 2011, Vujanovic et al. 2017). Although the mycoparasite showed efficacy in moderating DON, 3-ADON, 15-ADON, and production in Fusaria (Vujanovic and Chau 2012), the background mechanism of mycoparatism that occur at the molecular level is still unknown. Therefore, we hypothesized that SMCD 2220-01 effectiveness to reduce mycotoxin-producing capacities of Fusarium in co-culture is also related to its mycoparasitic ability to degrade or detoxify the substrates or media contaminated with Fusarium mycotoxins. The shifts in ZEN, DON, 3-ADON and 5-ADON was evaluated using Fusarium-standard thin layer chromatography (TLC) (Vujanovic et al. 2012) combined with high performance liquid chromatography-electrospray ionization-high resolution mass spectrometry (HPLC-ESI-HRMS) which is characterized with superior performance and sensitivity to discover masked or modified mycotoxins (De Boevre et al. 2016).

\section{Materials and methods}

\section{Fungal cultures, chemicals and media}

In this study, the mycoparasitic biocontrol Sphaerodes mycoparasitia SMCD 2220-01 strain deposited in IDAC under accession number 301008-01 (Public Health Agency of Canada-International Depositary Authority of Canada Collection, Winnipeg, Canada) has been used for decomposition and detoxification of Fusarium mycotoxins. Zearalenone (ZEN), deoxynivalenol (DON), 3-acetyl-deoxynivalenol (3-ADON), and 15-acetyl-deoxynivalenol (15-ADON) shown in Fig. 1 were purchased from Sigma-Aldrich Canada Ltd., Oakville, ON. HPLC grade organic solvents were purchased from Fisher Scientific. The stock solutions of each of mycotoxins were prepared by dissolving each mycotoxin in acetonitrile. Potato dextrose broth (PDB, BD Difco) and agar (PDA) were used for maintaining SMCD 2220-01 and biodegradation experiments.

\section{Evaluation of the efficacy of the mycotoxin-degrading capacity}

In order to induce degradation capacity of $S$. mycoparasitica, SMCD 2220-01 strain was grown on the cellulose membrane placed on PDA amended with $1 \mathrm{mg} \mathrm{L}^{-1}$ of each of mycotoxins. The mycelium of the induced SMCD 2220-01 was inoculated in $5 \mathrm{~mL}$ of PDB and incubated at

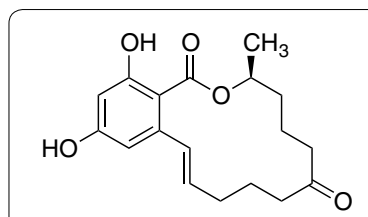

Zearalenone

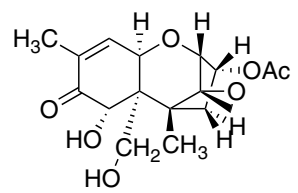

3-Acetyl-deoxynivalenol

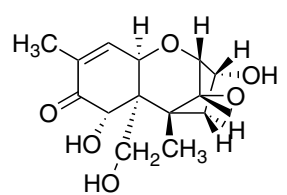

Deoxynivalenol

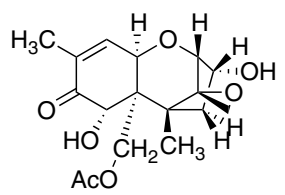

15-Acetyl-deoxynivalenol
Fig. 1 Chemical structure of the mycotoxin zearalenone (ZEN), deoxynivalenol (DON), 3-acetyl-deoxynivalenol (3-ADON), and 15-acetyldeoxynivalenol (15-ADON)

$23{ }^{\circ} \mathrm{C}$ on a rotary shaker at $120 \mathrm{rpm}$ for 3 days in the dark condition. The pre-cultured SMCD 2220-01 was incubated with $2 \mathrm{mg} \mathrm{L}^{-1}$ of ZEN, DON, 3-ADON, and 15-ADON, respectively. A non-treated with each mycotoxin and inoculated with SMCD 2220-01 in medium (only SMCD 2220-01) was used to exclude metabolites of SMCD 222001 . A treated with each mycotoxin but not inoculated with SMCD 2220-01 in medium (only each mycotoxin) was prepared to check natural decomposition of mycotoxins. A medium, PDB as a control was used to exclude impurities from the medium itself. All the cultures were incubated on a rotary shaker at $120 \mathrm{rpm}$ at $23^{\circ} \mathrm{C}$ in the dark condition. Cultures were harvested at 1,2 , and 3 weeks after the addition of mycotoxins. The harvested cultures were filtered by Whatman filter paper Grade 2 to remove mycelia. The culture filtrates were used for extraction of the residual mycotoxins by liquid-liquid partition.

\section{Detection and semi-quantification of mycotoxins by TLC}

The culture filtrates were extracted by $5 \mathrm{~mL}$ of ethyl acetate (EtOAc) and evaporated to dryness. The EtOAc extracts were dissolved in $200 \mu \mathrm{L}$ of chloroform for thin layer chromatography (TLC) (Bejaoui et al. 2006; GardaBuffon and Badiale-Furlong 2010; Teniola et al. 2005). For four mycotoxins, 4,8 , and $8 \mu \mathrm{L}$ of the final extracts of SMCD 2220-01, SMCD 2220-01 treated with mycotoxins, and mycotoxins in medium in sequence were spotted, along with authentic mycotoxin standards $(1 \mu \mathrm{L}$ of $1000 \mathrm{ppm}$ ) on the base of an aluminum TLC silica gel $60 \mathrm{~F}_{254}$ plate. The separation process was performed by using a mixture of dichloromethane and methanol with optimized developing times (95:5 for ZEN, 15-ADON, and 3-ADON and 93:7 for DON) as a mobile phase for developing the TLC plate (Abbas et al. 1984). Further, the developed TLC plate was dried and then visualized by 
charring solutions after checking under ultra violet light if needed. To interpret TLC spots, the relative mobility or retention factor $\left(R_{f}\right)$ was calculated by the following equation (Eq. 1).

$$
\begin{aligned}
\mathrm{R}_{\mathrm{f}}= & \text { Distance from start to center of substance spot } \\
& / \text { distance from start to solvent front }
\end{aligned}
$$

Semi-quantification of TLC spots for residual mycotoxins was achieved through densitometry analysis using Image J software. Image J software is available online at http://rsbweb.nih.gov/ij/plugins/index.html and provides an easy access to extract the area occupied by a specific color. Data are the mean of three replicate with error bars representing standard deviation analyzed by one-way analysis of variance (ANOVA) Tukey's HSD $(P<0.05)$. Percent degradation of the mycotoxins by SMCD 222001 was calculated by using the equation (Eq. 2).

$$
\text { Degradation rate }(\%)=\left(1-A / A_{\mathrm{C}}\right) \times 100(\%)
$$

where $A$ is the area of residual mycotoxin in the samples and $A_{\mathrm{c}}$ is the area of mycotoxin in the control (mycotoxin in medium).

\section{Confirmation of mycotoxin quantification by HPLC-ESI- HRMS}

In order to confirm the quantification of residual mycotoxins and to elucidate transformants of mycotoxins by SMCD 2220-01, HPLC-ESI-HRMS was performed on an Agilent 1100 series high-performance liquid chromatography (HPLC) system equipped with an automatic injector, quaternary pump, degasser, and a diode array detector (DAD, wavelength range 190-600 nm) connected to a Qstar XL systems Mass Spectrometer (Hybrid Quadruple-TOF LC/MS) with turbospray electrospray ionization (ESI) source. Chromatographic separations were carried out using Eclipse XDB-C-18 column (5 $\mu \mathrm{m}$ particle size silica, $150 \times 4.6 \mathrm{~mm}$ I.D.). All the extracted samples were dissolved in acetonitrile. Authentic mycotoxins $(2,4,8$, and $10 \mu \mathrm{L}$ of $100 \mathrm{ppm})$ were used for generation of standard curves, as well as confirmation of ionization patterns of each mycotoxin for detection.

The mobile phase consisted of a linear gradient of $0.1 \%$ formic acid in water and 0.1\% formic acid in methanol (95:5 in $5 \mathrm{~min}$, to $80: 20$ in $25 \mathrm{~min}$, to $50: 50$ in $35 \mathrm{~min}$, to $25: 75$ in

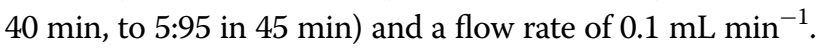
Data acquisition was carried out either positive or negative polarity mode for LC run (DON, 3-ADON, and ZEN on negative mode and 15-ADON on positive mode). Data processing was conducted by Analyst QS Software. Percent degradation of the mycotoxins by SMCD 2220-01 was calculated by using the equation (Eq. 3).

$$
\text { Degradation rate }(\%)=\left(1-C / C_{\mathrm{C}}\right) \times 100(\%)
$$

where $C$ is the residual concentration of mycotoxin in the sample $\left(\mathrm{mg} \mathrm{L}^{-1}\right)$ and $C_{\mathrm{c}}$ is the concentration of mycotoxin ( $\mathrm{mg} \mathrm{L}^{-1}$ ) in the control (mycotoxin in medium).

\section{Statistical analysis}

Data are the mean of three replicate with standard deviation. One-way analysis of variance (ANOVA) Tukey's HSD was used to test whether each of residual mycotoxins in samples and controls based on TLC was significantly different $(P<0.05)$.

\section{Results}

\section{Thin layer chromatography (TLC)}

TLC analysis indicated the different level of residual mycotoxins and metabolites of extracts of culture filtrate derived from SMCD 2220-01 at 1, 2, and 3 weeks old cultures after the addition of each mycotoxin (data at 3 weeks shown in Fig. 2). $R_{f}$ value of ZEN, 15-ADON, and
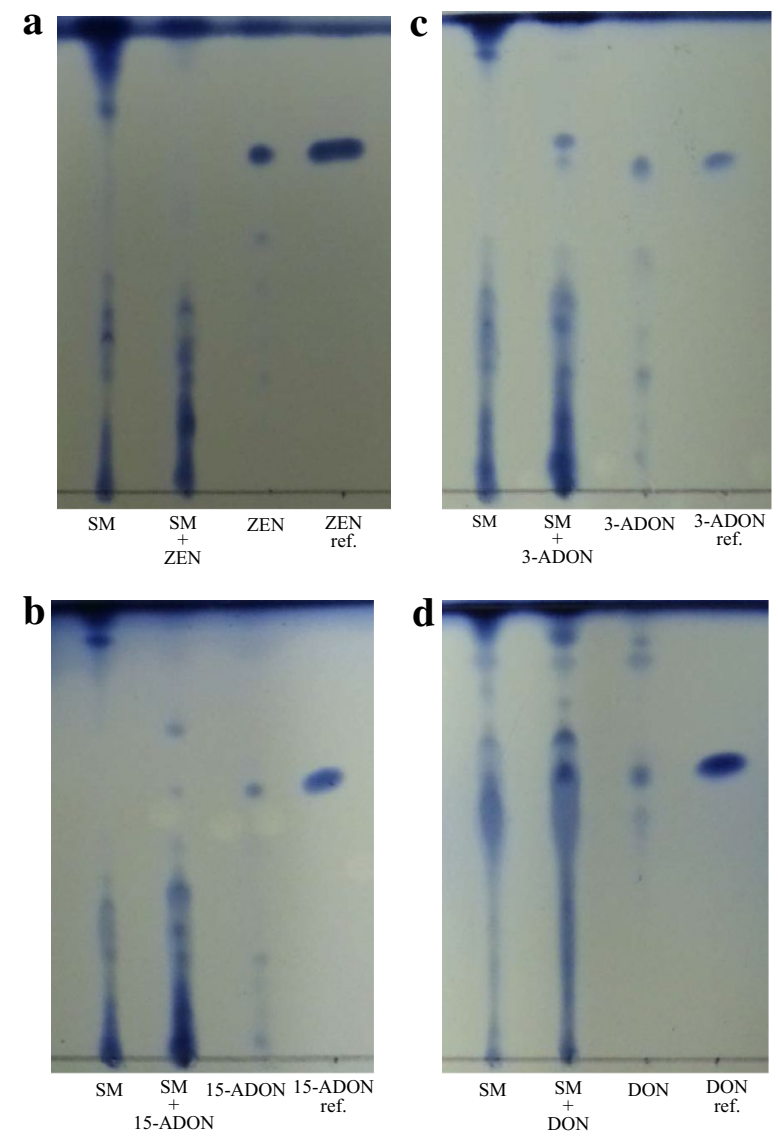

Fig. 2 Thin layer chromatograms of extracts of culture filtrates at 3 week incubation supplemented with $2 \mathrm{mg} \mathrm{L}^{-1}$ of ZEN (a), 15-ADON (b), 3-ADON (c), and DON (d). From the left side, each of lanes indicates the SMCD 2220-01 only, SMCD 2220-01 treated with each mycotoxin, each mycotoxin in medium, and authentic mycotoxin as a reference 
3 -ADON was $0.69,0.58$, and 0.68 in the solvent system (95\% dichloromethane and 5\% methanol with developing 2,4 , and 4 times), respectively. 15-ADON and 3-ADON showed transformants as separate spots. $R_{f}$ value of DON was 0.63 in the solvent system (93\% dichloromethane and $7 \%$ methanol with developing 5 times). It seemed that DON with the transformants by SMCD 2220-01 was masked due to the similar polarity between DON and the transformants.

Based on spot areas at $R_{f}$ value for each mycotoxin, densitometry analysis allowed us to check efficacy of SMCD $2220-01$ to decrease in ZEN by $38,100,100 \%$, 15 -ADON by $37,45,74 \%, 3-A D O N$ by $37,58,63 \%$ at 1,2 , and 3 weeks after the addition of each mycotoxin, respectively (Fig. 3A-C). However, DON could not be analyzed by TLC due to the similar polarity between DON and the transformants or the metabolites (Fig. 3D). In overall, through TLC, SMCD 2220-01 showed the most effective degradability on ZEN among other mycotoxins. In addition to ZEN, HPLC-ESI-HRMS analysis revealed also DON degradation products.

\section{HPLC-ESI-HRMS analysis}

Mycotoxin detection and quantification as well as qualification of residual mycotoxins in extracts of culture filtrate for each mycotoxin treatment exposed to SMCD 2220-01 were confirmed by extracted ion chromatograms (XIC) through HPLC-ESI-HRMS analysis. It was shown that SMCD 2220-01 degrades 97, 72, 58, and 89\% of ZEN, 15-ADON, 3-ADON, and DON at 3 weeks incubation in PDB after the addition of the mycotoxins, respectively (Fig. 4). The trend of mycotoxins degradation ability of SMCD 2220-01 indicated by LC-MS was fairly similar to that by TLC analysis, except for DON. In case of DON, $\mathrm{XIC}$ allowed us to calculate residual DON successfully and overcome TLC limitations.

The extract of ZEN in PDB showed a peak of $[\mathrm{M}-\mathrm{H}]^{-}$at $m / z 317.1480$ identified as ZEN, while the extract of culture filtrate of SMCD 2220-01 treated with ZEN in PDB showed a peak at $m / z 397.1052$ of in negative-ion mode (Fig. 5a, b). The ZEN degradation product, $\left[\mathrm{M}-\mathrm{H}+\mathrm{SO}_{3}\right]^{-}$at $\mathrm{m} / z 397.1052$ compound was identified as detoxified compound $\left(\mathrm{C}_{18} \mathrm{H}_{21} \mathrm{O}_{8} \mathrm{~S}\right)^{-}$ according to Plasencia and Mirocha (1991). The difference of mass unit (only 9.0 millimass) between the observed mass (397.1052) and calculated mass (397.0962) may result from the electron-withdrawing effect of the sulfate group and by affected by dissolving solvent (Barron et al. 1988). It is likely that two peaks at $m / z 195.0549$ and 117.0214 are related with PDB

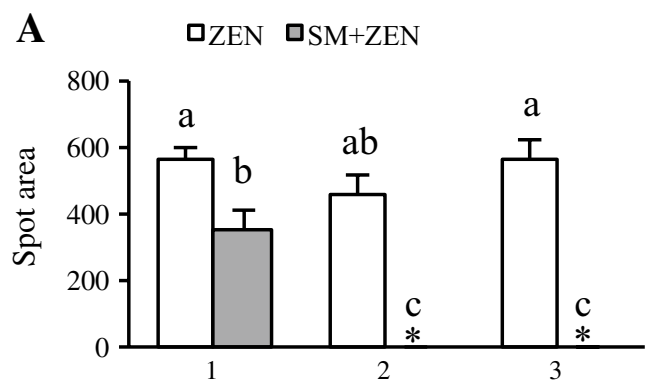

Time (week)

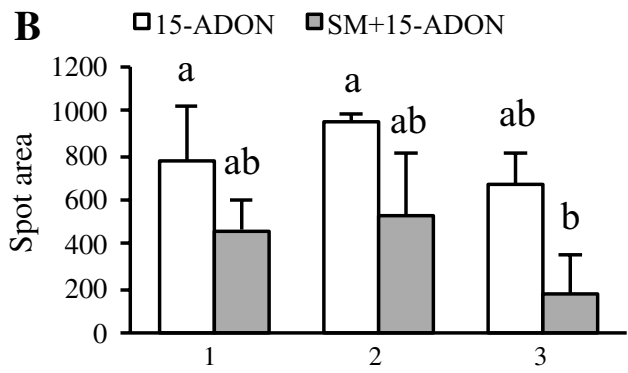

Time (week)
C a3-ADON aSM+3-ADON

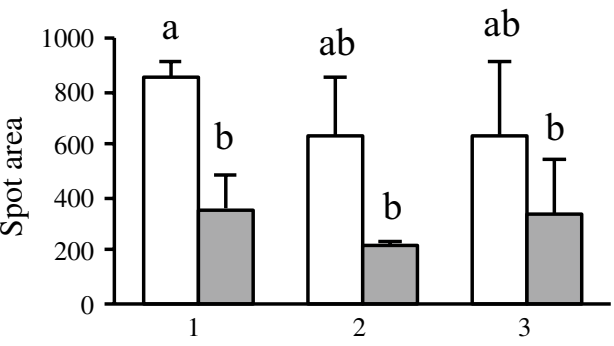

Time (week)

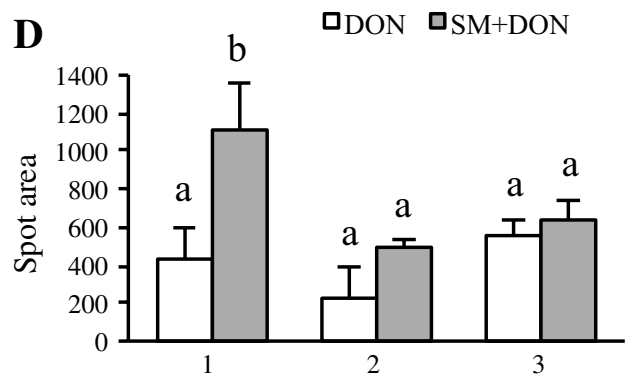

Time (week)

Fig. 3 Densitometry analysis for the residual mycotoxin levels at 1, 2, and 3 weeks after addition of each mycotoxin based on spot area of TLC. Data are the mean of three replicate with error bars representing standard deviation analyzed by ANOVA Tukey's HSD $(P<0.05)$. The same letters above the error bars do not differ significantly at $P<0.05$. ZEN $(\mathbf{A}), 15-\mathrm{ADON}(\mathbf{B}), 3-\mathrm{ADON}(\mathbf{C})$, and DON (D). Each mycotoxin in medium open square; SMCD 2220-01 with each mycotoxin in medium closed square; ${ }^{*}$ no residual ZEN 


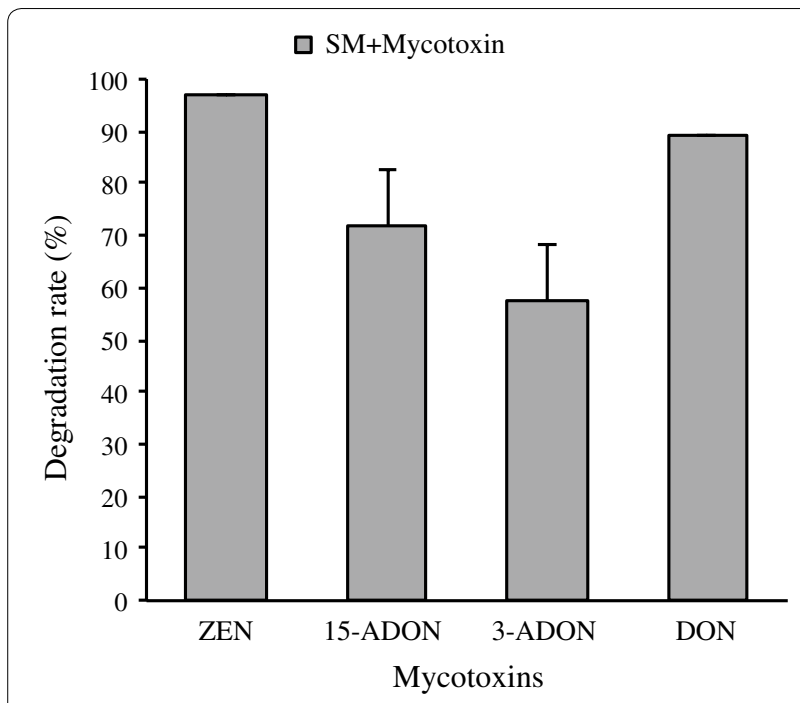

Fig. 4 Degradation rate (\%) of ZEN, 15-ADON, 3-ADON, and DON by SMCD 2220-01 at 3 weeks incubation. Values are the mean of three replicate and standard deviation

compositions (Fig. 5c). The peaks at $m / z 137.0268$ and 165.0582 seem to relate with metabolite(s) of SMCD 2220-01 (Fig. 5d).
The extracts of culture filtrates from both DON in PDB and SMCD 2220-01 treated with DON in PDB showed peaks of $[\mathrm{M}+\mathrm{HCOO}]^{-}$at $\mathrm{m} / z 341.1214$ and 341.1358 in negative-ion mode (Fig. 6a, b), identified as DON. It may be that peaks at $m / z 151.0214,237.0730$, and 345.2300 are related with DON degradation product by SMCD 2220-01 or its fragment or metabolite of SMCD 2220-01 induced by DON (Fig. 6b). Especially, a peak at $m / z 345.2300$ might be considered, as a fragment ion which lost $\mathrm{CH}_{2} \mathrm{O}$ from $\mathrm{CH}_{2} \mathrm{OH}$ group attached to the carbon at the C-6 position of deoxynivalenol-3-sulfate (Warth et al. 2014).

The extracts of culture filtrates from both 3-ADON in PDB and SMCD 2220-01 treated with 3-ADON in PDB showed a peak of $[\mathrm{M}+\mathrm{HCOO}]^{-}$at $\mathrm{m} / z 383.1345$ in negative-ion mode (Fig. 6c, d), identified as 3-ADON. It is likely that peaks at $\mathrm{m} / z 151.0214,273.1736$, and 345.2300 are related with $3-\mathrm{ADON}$ degradation products by SMCD 2220-01 or its fragments or metabolite of SMCD 2220-01 induced by 3-ADON (Fig. 6d). A peak at $\mathrm{m} / \mathrm{z} 345.2300$ might be a fragment ion of the deoxynivalenol-3-sulfate, which may be result of deacetylation of 3 -ADON (converted to DON) and then sulfation of DON by SMCD 2220-01.

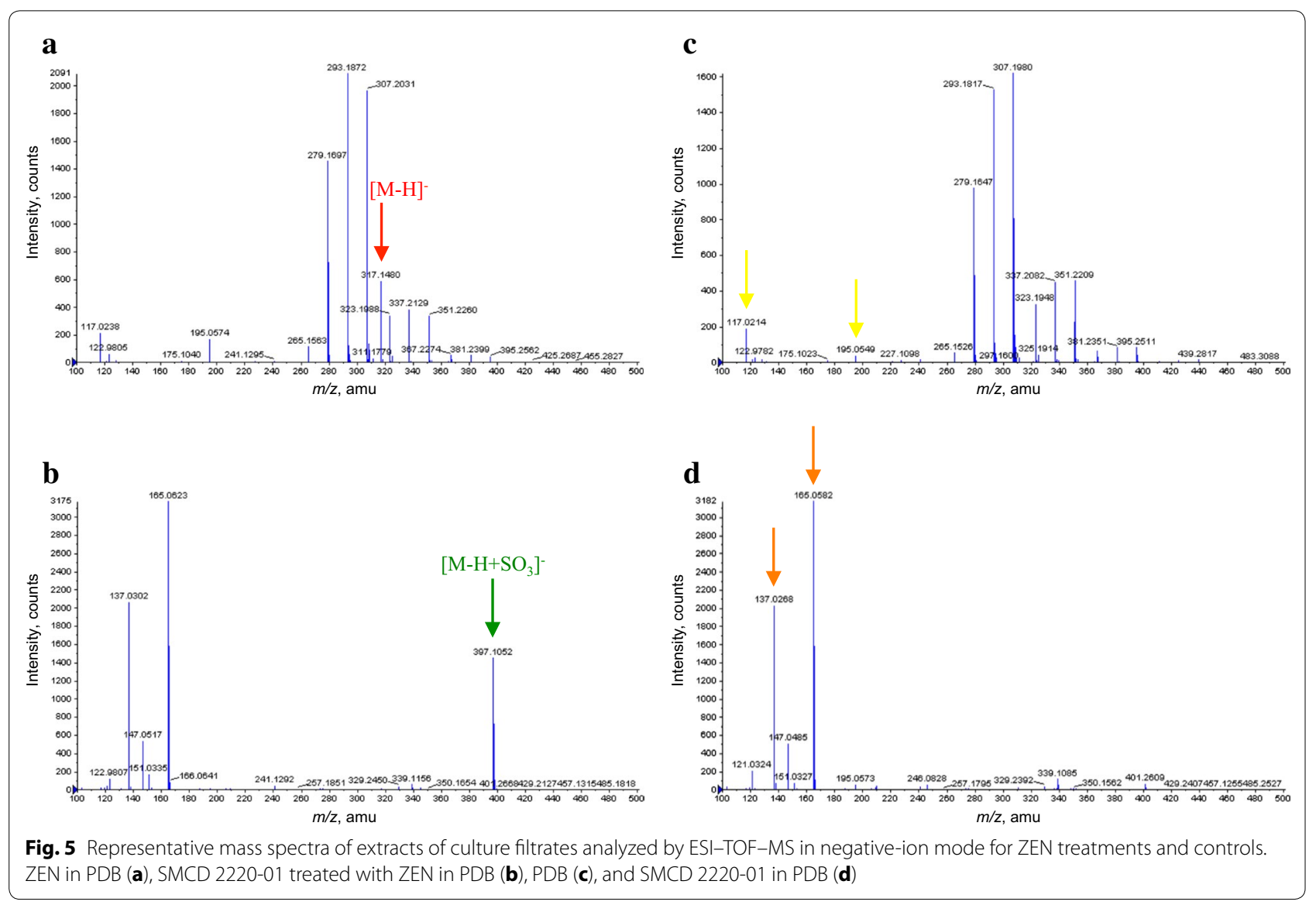



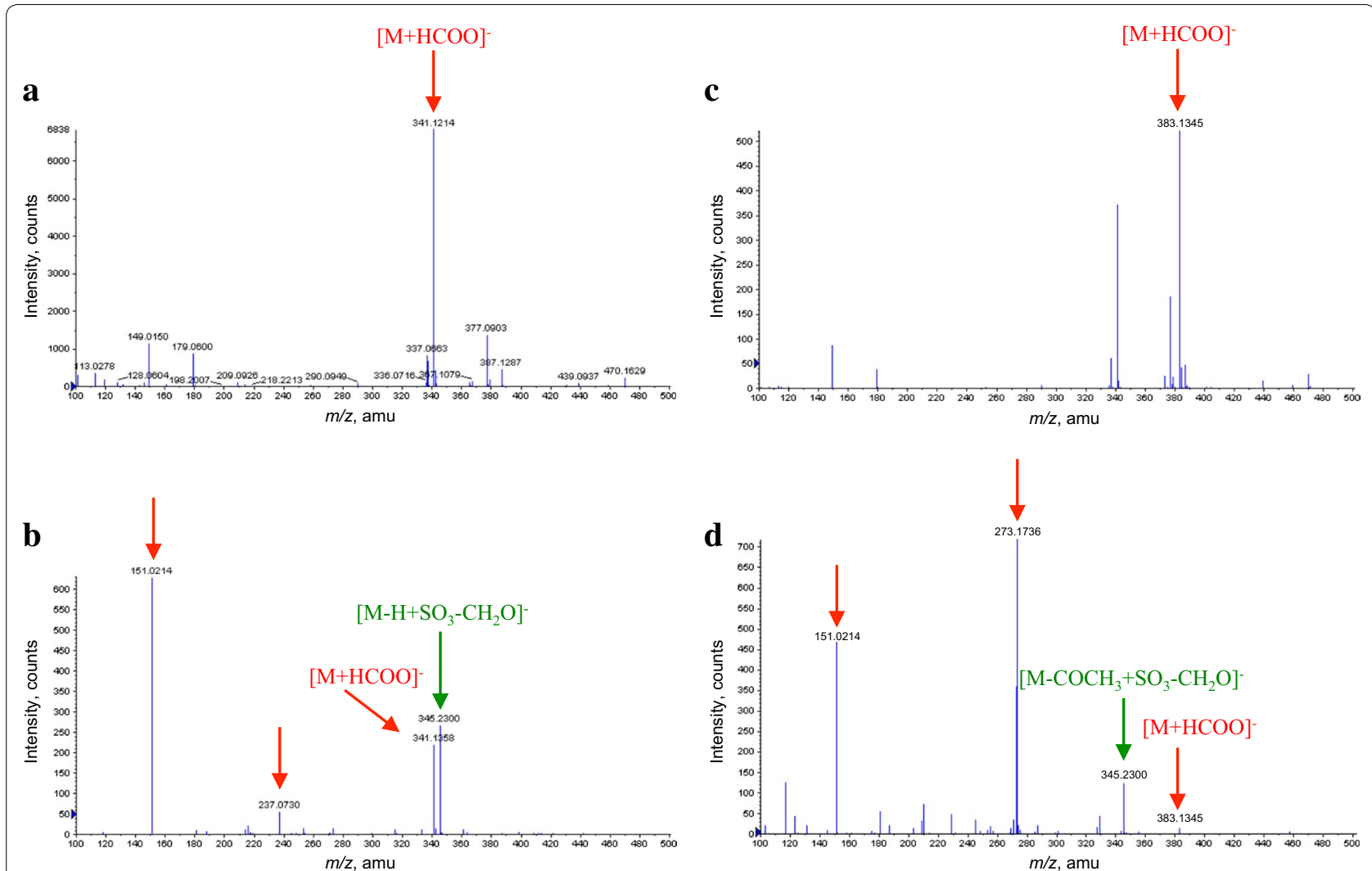

Fig. 6 Representative mass spectra of extracts of culture filtrates analyzed by ESI-TOF-MS in negative-ion mode for DON and 3-ADON treatments. DON in PDB (a), SMCD 2220-01 treated with DON in PDB (b), 3-ADON in PDB (c), and SMCD 2220-01 treated with 3-ADON in PDB (d)

The extracts culture filtrates of both 15-ADON in PDB and SMCD 2220-01 treated with 15-ADON in PDB showed peaks of $[\mathrm{M}+\mathrm{H}]^{+}$at $m / z 339.1603$ and $[\mathrm{M}+\mathrm{Na}]^{+}$at $m / z 361.1453$ in positive-ion mode (Fig. 7a, b), identified as $15-\mathrm{ADON}$. It is likely that peaks at $\mathrm{m} / z 225.2032$ are related with 15-ADON, which might be fragments of $15-\mathrm{ADON}$. The very weak peak at $\mathrm{m} / z 190.0598$ seems to relate with 15 -ADON degradation product by SMCD 2220-01. The peaks at $m / z 151.0791$ and 214.9233 seem to relate with metabolite(s) of SMCD 2220-01 compared with PDB (Fig. 7c, d).

\section{Discussion}

The presence of mycotoxins is inherent to many grains, food and feed products worldwide (Vanhoutte et al. 2016). For many years the research community focused on the occurrence of singular mycotoxins but nowadays scientific interest shifts to studies involving multiple mycotoxins, in particularly for Fusarium species and associated mycotoxins in cereal grain, food and feed (Kim and Vujanovic 2016). Microbial detoxification or biotransformation of mycotoxins includes different types of reaction, such as acetylation, glucosylation, ring cleavage, hydrolysis, deamination, and decarboxylation (McCormick 2013).

Biotransformation or biodegradation of ZEN has been reported by Bacillus spp. (Xu et al. 2016) and Pseudomonas spp. strains (Tan et al. 2014). However, metabolic products of ZEN were not identified from those strains. A possible pathway might comprise of cleavage of a ring structure followed by decarboxylation for complete degradation of ZEN as shown by Bacillus strains (Tinyiro et al. 2011). Besides bacteria, several fungi were known as degrader of ZEN by producing different metabolites of ZEN: Rhizopus spp. producing $\alpha$-zearalenol and $\beta$-zearalenol (Brodehl et al. 2014); Aspergillus ochraceous and Aspergillus niger producing $\alpha$-zearalanol and $\beta$-zearalanol (El-Sharkawy and Abul-Hajj 1988b); Cunninghamella bainieri producing 2,4-dimethoxyzearalenone and 2-methoxyzearalenone (El-Sharkawy and Abul-Hajj 1988b); Rhizopus arrhizus producing zearalenone 4-sulfate (El-Sharkaway et al. 1991); Thamidium elegans and Mucor bainieri producing zearalenone-4- $\beta$ D-glucoside (El-Sharkawy and Abul-Hajj 1987). However, some of ZEN metabolic products such as $\alpha$-zearalenol, $\alpha$-zearalanol, and $\beta$-zearalanol showed higher toxicity 

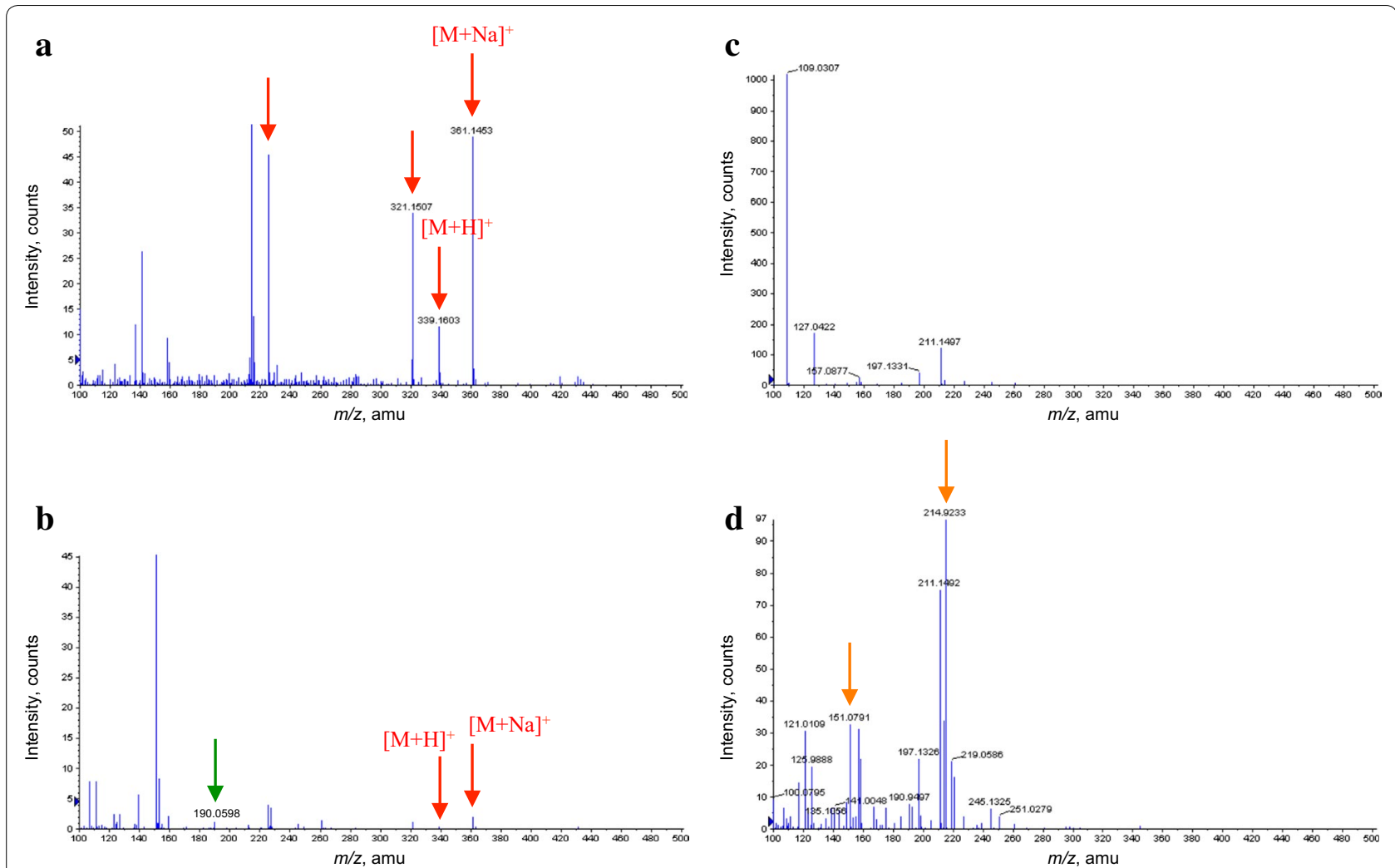

Fig. 7 Representative mass spectra of extracts culture filtrates analyzed by ESI-TOF-MS in positive-ion mode for 15-ADON treatments and controls. 15-ADON in PDB (a), SMCD 2220-01 treated with 15-ADON in PDB (b), PDB (c), and SMCD 2220-01 in PDB (d)

and higher relative estrogenicity than ZEN (Shier et al. 2001).

Divergently, a fungal mycoparasite Clonostachys rosea showed the ability to detoxify ZEN to a ring cleavage product, 1-(3,5-dihydroxy-phenyl)-10'-hydroxy-1'Eundecene-6'-one (Kakeya et al. 2002; Takahashi-Ando et al. 2002). Furthermore, it was shown that the detoxification of ZEN by zearalenone hydrolase of $C$. rosea is crucial for the successful mycoparasitism against $F$. graminearum (Kosawang et al. 2014).

In this study, S. mycoparasitica SMCD 2220-01 was efficient in decreasing the level of multiple Fusarium mycotoxins including ZEN by $97 \%$, DON by $89 \%$, $15-\mathrm{ADON}$ by $72 \%$, and $3-\mathrm{ADON}$ by $58 \%$ revealed by TLC and HPLC-ESI-HRMS. The transformant of ZEN by SMCD 2220-01 could be identified as zearalenone sulfate as revealed by LC-ESI-HRMS analysis (Fig. 8a). Furthermore, deoxynivalenol sulfate as transformed DON and 3-ADON metabolic product by SMCD 2220-01 was also detected (Fig. 8b). These findings demonstrate that the mycoparasite not only parasites on the host, but also degrades Fusarium mycotoxins or transforms them to less toxic compounds. Indeed, it was reported that modification or transformation of the aromatic ring of ZEN resulted in a remarkably decreased estrogenic activity compared to non-transformed ZEN (Drzymala et al. 2015). Therefore, biodegradation of the mycotoxins by SMCD 2220-01 could be considered as a process of detoxification for ZEN and DON, minimazing Fusarium mycotoxins in field crops and preventing reduction in grade and end-use quality of grains, food and feed.

Zearalenone sulfate, as a ZEN degradation product by SMCD 2220-01, is different from the ZEN degradation product by $C$. rosea. The differences in a type of degradation products may be due to distinct extracellular enzymes employed. In order to illuminate the metabolic mechanisms of degradation and/or detoxification of ZEN and DON by SMCD 2220-01, more research should be done on the specific fungal enzymes and their abilities to biotransform Fusarium mycotoxins to less toxic or non-toxic metabolites (Kluger et al. 2015; Ji et al 2016). The expression of genes coding for degrading enzymes involved in reduction of mycotoxins, are merited. It is important to note a comparative advantage of SMCD 2220-01 properties, as specific-mycoparasite to mycotoxigenic Fusaria, as source of the enzymes and vital biotechnological tool to mycotoxin biodegradation and biodetexofication (Kim and Vujanovic 2016). The 


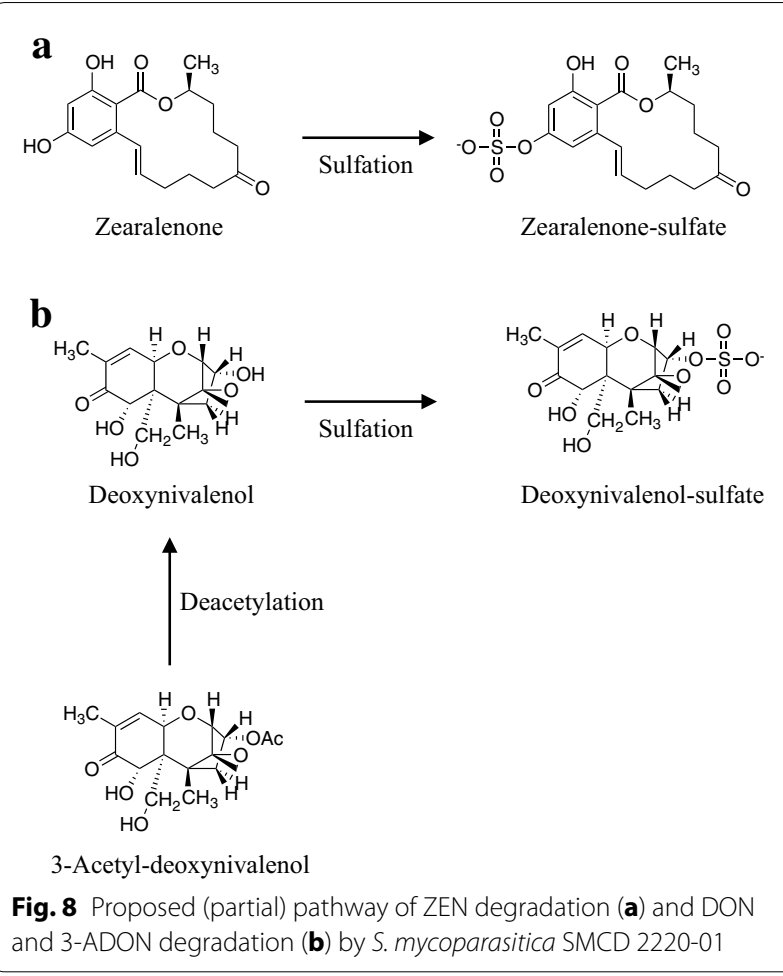

Sphaerodes mycoparasitic properties might be tightly connected to its biotrophic mycoparasite lifestyle and resistance to Fusarium, which implies that SMCD 222001 could be used as an efficient biocontrol agent. A full understanding and appropriate application of this mycoparasite should be helpful towards the development of novel microbiological solutions for reducing mycotoxin contamination-related to Fusarium infection in grains and for increasing mycotoxin detoxification in foods and feeds.

\section{Abbreviations}

AUR: aurofusarin; 3-ADON: 3-Acetyldeoxynivalenol; 15-ADON: 15-Acetyldeoxynivalenol; DON: deoxynivalenol; HPLC-ESI-HRMS: high performance liquid chromatography-electrospray ionization-high resolution mass spectrometry; PDB: potato dextrose broth; TLC: thin layer chromatography; ZEN: zearalenone.
\end{abstract}

\section{Authors' contributions}

The authors contributed equally to this article. Both authors read and approved the final manuscript.

\section{Acknowledgements}

This research was financially supported by Natural Sciences and Engineering Research Council of Canada_Discovery Grant (405823) to Dr. V. Vujanovic.

\section{Competing interests}

The authors declare that they have no competing interests.

\section{Availability of data and materials}

The datasets supporting the conclusions of this article are included within the main text and additional files or Figs. 1, 2, 3, 4, 5, 6, 7, and 8 .

\section{Funding}

This work was supported by Natural Sciences and Engineering Research

Council of Canada fund for promoting Discovery Science in Canada.

\section{Publisher's Note}

Springer Nature remains neutral with regard to jurisdictional claims in published maps and institutional affiliations.

Received: 22 June 2017 Accepted: 28 June 2017

Published online: 06 July 2017

\section{References}

Abbas HK, Mirocha CJ, Shier WT (1984) Mycotoxins produced from fungi isolated from foodstuffs and soil: comparison of toxicity in fibroblasts and rat feeding tests. Appl Environ Microbiol 48(3):654-661

Barron D, Varin L, Ibrahim RK, Harborne JB, Williams CA (1988) Sulphated flavonoids - an update. Phytochemistry 27(8):2375-2395. doi:10.1016/0031-9422(88)87003-1

Bejaoui H, Mathieu F, Taillandier P, Lebrihi A (2006) Biodegradation of ochratoxin A by Aspergillus section Nigri species isolated from French grapes: a potential means of ochratoxin A decontamination in grape juices and musts. FEMS Microbiol Lett 255(2):203-208. doi:10.1111/j.1574-6968.2005.00073.x

Brodehl A, Moller A, Kunte HJ, Koch M, Maul R (2014) Biotransformation of the mycotoxin zearalenone by fungi of the genera Rhizopus and Aspergillus. FEMS Microbiol Lett 359(1):124-130. doi:10.1111/1574-6968.12586

Caldwell RW, Tuite J, Stob M, Baldwin R (1970) Zearalenone production by Fusarium species. Appl Microbiol 20(1):31-34

De Boevre M, Ediage EN, Van Poucke C, De Saeger (2016) Masked mycotoxins in food: formation, occurence and toxicological relevance. In: C. Dall'Asta, F. Berthiller (ed) Chapter 4: In Masked mycotoxins in food: formation, occurrence and toxicological relevance. pp. 50-72. doi: 10.1039/9781782622574-00050

Drzymala SS, Binder J, Brodehl A, Penkert M, Rosowski M, Garbe LA, Koch M (2015) Estrogenicity of novel phase I and phase II metabolites of zearalenone and cis-zearalenone. Toxicon 105:10-12. doi:10.1016/j. toxicon.2015.08.027

Ehrlich KC, Daigle KW (1987) Protein synthesis inhibition by 8-oxo-12,13-epoxytrichothecenes. Biochim Biophys Acta 923(2):206-213

El-Sharkaway SH, Selim MI, Afifi MS, Halaweish FT (1991) Microbial transformation of zearalenone to a zearalenone sulfate. Appl Environ Microbiol 57(2):549-552

El-Sharkawy S, Abul-Hajj Y (1987) Microbial transformation of zearalenone, I. Formation of zearalenone-4-O- $\beta$-glucoside. J Nat Products 50(3):520-521. doi:10.1021/np50051a038

El-Sharkawy S, Abul-Hajj YJ (1988a) Microbial cleavage of zearalenone: the fate of foreign compounds in biological systems. Xenobiotica 8(4):365-371. doi:10.3109/00498258809041672

El-Sharkawy SH, Abul-Hajj YJ (1988b) Microbial transformation of zearalenone. 2. Reduction, hydroxylation, and methylation products. J Org Chem 53(3):515-519. doi:10.1021/jo00238a008

Garda-Buffon J, Badiale-Furlong E (2010) Kinetics of deoxynivalenol degradation by Aspergillus oryzae and Rhizopus oryzae in submerged fermentation. J Braz Chem Soc 21:710-714

Hussein HS, Brasel JM (2001) Toxicity, metabolism, and impact of mycotoxins on humans and animals. Toxicology 167(2):101-134. doi:10.1016/ S0300-483X(01)00471-1

labal SZ, Asi MR, Jinap S, Rashid U (2014) Detection of aflatoxins and zearalenone contamination in wheat derived products. Food Control 35(1):223-226. doi:10.1016/j.foodcont.2013.06.048

Ji C, Fan Y, Zhao L (2016) Review on biological degradation of mycotoxins. Anim Nutr 2:127-133

Kakeya H, Takahashi-Ando N, Kimura M, Onose R, Yamaguchi I, Osada H (2002) Biotransformation of the mycotoxin, zearalenone, to a non-estrogenic compound by a fungal strain of Clonostachys sp. Biosci Biotech Biochem 66(12):2723-2726. doi:10.1271/bbb.66.2723

Katzenellenbogen BS, Katzenellenbogen JA, Mordecai D (1979) Zearalenones: characterization of the estrogenic potencies and receptor interactions of a series of fungal $\beta$-resorcylic acid lactones. Endocrinology 105(1):33-40 
Kim SH, Vujanovic V (2016) Relationship between mycoparasites lifestyles and biocontrol behaviors against Fusarium spp. and mycotoxins production. Appl Microbiol Bio 100(12):5257-5272. doi:10.1007/s00253-016-7539-z

Kluger B, Bueschl C, Lemmens M, Michlmayr H, Malachova A, Koutnik A, Maloku I, Berthiller F, Adam G, Krska R, Schuhmacheret R (2015) Biotransformation of the mycotoxin deoxynivalenol in Fusarium resistant and susceptible near isogenic wheat lines. PLoS ONE 10(3):e0119656. doi:10.1371/journal.pone.011965

Kosawang C, Karlsson M, Vélëz H, Rasmussen PH, Collinge DB, Jensen B, Jensen DF (2014) Zearalenone detoxification by zearalenone hydrolase is important for the antagonistic ability of Clonostachys rosea against mycotoxigenic Fusarium graminearum. Fungal Biol 118(4):364-373. doi:10.1016/j. funbio.2014.01.005

McCormick SP (2013) Microbial detoxification of mycotoxins. J Chem Ecol 39(7):907-918. doi:10.1007/s10886-013-0321-0

Middlebrook JL, Leatherman DL (1989) Binding of T-2 toxin to eukaryotic cell ribosomes. Biochem Pharmacol 38(18):3103-3110

Plasencia J, Mirocha CJ (1991) Isolation and characterization of zearalenone sulfate produced by Fusarium spp. Appl Environ Microbiol 57(1):146-150

Shier WT, Shier AC, Xie W, Mirocha CJ (2001) Structure-activity relationships for human estrogenic activity in zearalenone mycotoxins. Toxicon 39(9):1435-1438. doi:10.1016/S0041-0101(00)00259-2

Sundstøl Eriksen G, Pettersson H, Lundh T (2004) Comparative cytotoxicity of deoxynivalenol, nivalenol, their acetylated derivatives and deepoxy metabolites. Food Chem Toxicol 42(4):619-624. doi:10.1016/j. fct.2003.11.006

Takahashi-Ando N, Kimura M, Kakeya H, Osada H, Yamaguchi I (2002) A novel lactonohydrolase responsible for the detoxification of zearalenone: enzyme purification and gene cloning. Biochem J 365(Pt 1):1-6. doi:10.1042/bj20020450

Tan H, Hu Y, He J, Wu L, Liao F, Luo B, He Y, Zuo Z, Ren Z, Zhong Z, Peng G, Deng J (2014) Zearalenone degradation by two Pseudomonas strains from soil. Mycotoxin Res 30(4):191-196. doi:10.1007/s12550-014-0199-x

Teniola OD, Addo PA, Brost IM, Färber P, Jany KD, Alberts JF, van ZyI WH, Steyn PS, Holzapfel WH (2005) Degradation of aflatoxin B1 by cell-free extracts of Rhodococcus erythropolis and Mycobacterium fluoranthenivorans sp. nov. DSM44556T. Int J Food Microbiol 105(2):111-117. doi:10.1016/j. ijfoodmicro.2005.05.004
Tinyiro SE, Wokadala C, Xu D, Yao W (2011) Adsorption and degradation of zearalenone by Bacillus strains. Folia Microbiol 56(4):321. doi:10.1007/ s12223-011-0047-8

Vanhoutte I, Audenaert K, De Gelder L (2016) Biodegradation of mycotoxins: tales from known and unexplored worlds. Front Microbiol 7:561. doi:10.3389/fmicb.2016.00561

Vujanovic V, Chau HW (2012) Monitoring Fusarium complex mycelia replacement by mycopathogenic Sphaerodes using alcohol percentage test, qRT-PCR and HPLC. Physiol Mol PI Path 80:28-34. doi:10.1016/j. pmpp.2012.07.004

Vujanovic V, Goh YK (2009) Sphaerodes mycoparasitica sp. nov., a new biotrophic mycoparasite on Fusarium avenaceum, F. graminearum and F. oxysporum. Mycol Res 113:1172-1180. doi:10.1016/j.mycres.2009.07.018

Vujanovic V, Goh YK (2010) Sphaerodes mycoparasites and new Fusarium hosts for S. mycoparasitica. Mycotaxon 114(1):179-191

Vujanovic V, Goh YK (2011) Sphaerodes mycoparasitica biotrophic mycoparasite of 3-acetyldeoxynivalenol- and 15-acetyldeoxynivalenol-producing toxigenic Fusarium graminearum chemotypes. FEMS Microbiol Lett 316(2):136-143. doi:10.1111/j.1574-6968.2010.02201.x

Vujanovic V, Goh YK, Daida P (2012) Heat- and cold-shock responses in Fusarium graminearum 3 acetyl- and 15 acetyl-deoxynivalenol chemotypes. J Microbiol 50(1):97-102. doi:10.1007/s12275-012-1381-5

Vujanovic V, Daida MA, Daida P (2017) qPCR assessment of aurofusarin gene expression in mycotoxigenic Fusarium species challenged with mycoparasitic and chemical control agents. Biol Control 109:51-57. doi:10.1016/j. biocontrol.2017.03.010

Warth B, Fruhmann P, Wiesenberger G, Kluger B, Sarkanj B, Lemmens M, Hametner C, Frohlich J, Adam G, Krska R, Schuhmacher R (2014) Deoxynivalenol-sulfates: identification and quantification of novel conjugated (masked) mycotoxins in wheat. Anal Bioanal Chem. doi:10.1007/ s00216-014-8340-4

Xu J, Wang H, Zhu Z, Ji F, Yin X, Hong Q, Shi J (2016) Isolation and characterization of Bacillus amyloliquefaciens ZDS-1: exploring the degradation of Zearalenone by Bacillus spp. Food Control 68:244-250. doi:10.1016/j. foodcont.2016.03.030

Zhou T, He J, Gong J (2008) Microbial transformation of trichothecene mycotoxins. World Mycotoxin J 1(1):23-30. doi:10.3920/WMJ2008.x003

\section{Submit your manuscript to a SpringerOpen ${ }^{\circ}$ journal and benefit from:}

- Convenient online submission

- Rigorous peer review

- Open access: articles freely available online

- High visibility within the field

- Retaining the copyright to your article

Submit your next manuscript at $\boldsymbol{\nabla}$ springeropen.com 